patient complaining of great pain above the right breast and difficulty in breathing) a colourless frothy liquid, at times slightly stained with hæmoglobin, had been coughed up in considerable quantities. There was about half a pint in all. Of specific gravity about 1030, it coagulated on boiling, contained no red corpuscles when examined microscopically, but gave the spectroscopic test for oxyhæmoglobin. As the more alarming symptoms seemed to be due to an acute engorgement of the right ventricle a small venesection was performed, about ten ounces of blood being withdrawn, and five minims of solution of strychnine, together with 20 minims of ether purus, were given hypodermically, weak brandy and water being ordered by the mouth, in teaspoonful doses, as the respiratory distress rendered swallowing very difficult.

The patient's condition having undergone a slight improvement 1 left her about 4 A.M., givin $\ddot{\circ}$ instructions that the hypodermic injections should be continued as directed and that I should be sent for immediately if any change for the worse took place. To my surprise I heard no more of the patient until I made my usual visit to the ward on the following morning at 10 o'clock. She was then resting quietly; all signs of dyspncea had disappeared and, except for some facial pallor and slight exhaustion, she seemed to be none the worse for her experience of the early hours. On examination of the chest a few moist râles conld still be heard over the affected area while all signs of cardiac dilatation had vanished and at 4 o'clock on the same afternoon the chest, being again examined, this time in company with one of my colleagues, was pronounced by him to be normal. From this time onwards the puerperium was uneventful, save for the development of a small, superficial mammary abscess which rapidly yielded to appropriate treatment, and the patient was allowed up on the twenty-first day and discharged herself from the infirmary two days later.

As regards a diagnosis, dyspnoea coming on suddenly after labour in a healthy woman such as this patient, taken together with the physical signs present during the attack, seemed to me to point to an embolus of uterine origin which had been carried away and become arrested in the pulmonary circulation, while the fact of the signs clearing up so rapidly (all physical signs had disappeared 16 hours after onset) makes it probable that the embolus, if such it were, must have been composed of air rather than dislodged blood clot. If, however, this diagnosis be admitted as possibly a correct one it seems difficult to imagine how the air was introduced into the uterus, since no intra-uterine manipulations were made nor was a douche given either before or after labour. The point about which I feel most interest is as to whether a hurried expulsion of the afterbirth, such as was effected in this case, could have given rise to the condition described, air presumably being sucked into the vagina as the uterus was released from the somewhat powerful grasp necessary to expel a not sufficiently separated placenta; and if this be so, why so lengthy an interval as an hour elapsed before the onset of the disquieting symptoms? Should any of your readers feel sufficiently interested I should be grateful for an expression of opinion upon this, to me, somewhat puzzling experience.

Cambridge Heath, N.t.

\section{Clinical antes:}

MEDICAL, SURGICAL, OBSTETRICAL, AND THERAPEUTICAL.

\section{A CASE OF FORWARD DISLOCATION OF THE RADIUS AND ULNA WITHOUT FRACTURE OF THE OLECRANON PROCESS OF THE ULNA.}

By F. W. Staunton, M.B. Dub.,

HONORARY SURGEON TO WATERFORD COUNTY AND CITY INFIRMARY.

THE patient in this case was a man, aged 45 years, whose description of his accident was to the effect that "while walking quickly downstairs he felt as if he had trod on something slippery; his foot went from under him, he twisted round in order to save himself and fell backwards downstairs, the tip of his left elbow striking the edge of the staircase first." On examination the elbow was seen to be held at an angle of $130^{\circ}$. It could be slightly flexed and extended and permitted a good deal of lateral movement. The head of the radius and the olecranon process of the ulna could be felt lying on the shaft of the humerus about two and a quarter inches above the condyles. It was possible to feel both condyles of the humerus, the olecranon fossa, the coronoid depression, the trochlea, and the capitellum; in fact, the lower end of the humerus could be easily grasped in the band. There was distinct lengthening of the forearm.

The points of interest in this case were: (1) its rarity (2) the absence of fracture of the olecranon; and (3) the maintenance of the relationship between the radius anc ulna. Attention has been called to this in Treves's "Surgery" by Mr.Marmaduke Sheild who states that "this dislocation is extremely rare," and quotes Canton's case in which the radius maintained its relationship to the ulna. Looking up the literature on the subject I find that there are about 12 recorded cases. Erichsen states that six cases of this dis. location are on record. Rose and Carless state that fracture of the olecranon always occurs in this dislocation. Gould and Warren in their "International Text-book of Surgery" refer to this dislocation as " rare and its occurrence without existing fracture has been doubted; in point of fact the latter complication has been still more rarely recorded." Helferich says: "This is a very rare injury ; it was formerly said it never occurred without simultaneous fracture of olecranon. May be produced by a fall or blow on olecranon while arm is in extreme flexion." Hamilton quotes nine cases and states "that it seems to have been in most cases caused by a fall upon elbow while forearm is forcibly flexed." Cheyne and Burghard state that this dislocation "is sometimes met with as result of a severe blow upon back of flexed elbow."

Stimson in his book refers to a case which was under his care in 1895. He says: "The elbow is held at a right angle but can be somewhat flexed and extended; is moveable laterally. The epitrochlea can be plainly felt, also the inner face and edge of trochlea. The overlying flexor muscles of hand having been torn away from humerus, the tip of olecranon is below, and even a little in front of, the trochlea, the inner anterior portion of articular surface of which can also be felt on depressing the skin. The head of radius in front of capitellum ; the forearm is markedly abducted. The only other reported case is Wright's. The symptoms were similar."

Waterford.

\section{DISAPPEARANCE OF RODENT ULCER UNDER THE} APPLICATION OF THE X RAYS.

By Frederick B. JEFFERISS, F.R.C.S. EDIs. M.R.C.S. ENG., L.R.C.P. LOND.

THE successful treatment of rodent ulcer by the application of the $\mathrm{x}$ rays is now well established and many cases are on record where a complete recovery has resulted. The same, however, cannot be said of carcinomata, some cases improving for a time but relapsing again or even getting worse as the treatment is continued.

The patient in the present instance was an elderly woman who suffered from an epithelial growth on the left frontal region. The history was to the effect that about two years before she first came under my notice she observed a small pimple at the site of the growth which she injured by accidently striking it against the mantelpiece causing ulceration. She treated it herself with zinc ointment without any benefit and it steadily grew for the two years. When I first saw her the growth was about as big as a fiveshilling piece, rounded in shape, and very much raised, the ulcer on the top of the growth being about half an inch above the level of the surrounding skin, so that the whole growth was crateriform in appearance. It was very vascular, several large veins ramifying on the surface, and bleeding was easily induced on manipulation. There was no pain but the growth was very tender and the whole was soft, including the edges of the ulcer, which although heaped up were not indurated; the mass was freely moveable and none of the neighbouring glands were involved. A diagnosis of rodent ulcer was made but it could not be confirmed as the patient refused to allow a piece to be removed for microscopic examination.

I began the treatment with the $\mathrm{x}$ rays on March 5th, 1904, giving two sittings a week of five minutes each. No change 